\section{Über den Einfluß der Dichte der Leitungs- elektronen eines Supraleiters auf seine Übergangstemperatur}

\author{
W. KESSEL
}

Physikalisch-Technische Bundesanstalt, Braunschweig

(Z. Naturforsch. 24 a, 294-295 [1969] ; eingeg. am 12. Dezember 1968)

Nach der BCS-Theorie ${ }^{1}$ geht in die Supraleiteigenschaften eines Metalles die Kopplungskonstante

$$
g=V D\left(\varepsilon_{\mathrm{F}}\right)
$$

wesentlich ein. Dabei ist $D\left(\varepsilon_{\mathrm{F}}\right)$ die Dichte der Elektronenzustände an der Fermi-Kante $\varepsilon_{\mathrm{F}}$ des normalleitenden Zustandes und $V$ die mittlere Wechselwirkungsenergie der Leitungselektronen. Neben der elektrostatischen Coulomb-Abstoßung existiert im Metall eine von den Phononen induzierte anziehende Kopplung der Elektronen, die im Supraleiter die Abstoßung überwiegt. Die Úbergangstemperatur $T_{\mathrm{c}}$ ist eine von der Kopplungskonstanten $g$ stark abhängende Funktion

$$
k_{\mathrm{B}} T_{\mathrm{c}}=1,14 \hbar \omega_{0} \exp (-1 / g) .
$$

Deshalb drückt sich jede Änderung von $g$ in einer entsprechenden Verschiebung der Übergangstemperatur aus. In (2) bedeutet $k_{\mathrm{B}}$ die Boltzmann-Konstante, $\hbar$ die Plancksche Konstante dividiert durch $2 \pi$ und $\omega_{0}$ eine mittlere Phononenfrequenz von der Größenordnung der Debye-Frequenz. Eine Änderung der Zahl der Leitungselektronen bewirkt daher über eine Änderung der Fermi-Energie $\varepsilon_{F}$ eine Verlagerung des Sprungpunktes des betreffenden Supraleiters. Außer durch Untersuchungen an supraleitenden Legierungen ${ }^{2}$ ist diese Verschiebung der Sprungtemperatur an dünnen Metallfilmen durch direkten Elektronenentzug beim elektrostatischen Aufladen ${ }^{3}$ und beim oberflächlichen Oxidieren ${ }^{4}$ nachgewiesen worden. Nach einer einfachen Analyse ${ }^{5}$ der Experimente ist die Kopplungskonstante $g$ eine monoton abnehmende Funktion der Zustandsdichte an der Fermi-Kante. Eine Erklärung hierfür haben Bardasis, Glover, Prange und YOrke ${ }^{6}$ gegeben. Sie erkannten, daß bei der von den Phononen induzierten anziehenden Wechselwirkung nur Impulse, die kleiner oder etwa gleich dem Debye-Impuls sind, zwischen den Elektronen ausgetauscht werden können. Dadurch wird der Wechselwirkungsparameter proportional zu $D\left(\varepsilon_{\mathrm{F}}\right)^{-2}$. Für diese Beschreibung wird jedoch von den Autoren nur der durch die Phononen vermittelte Anteil der Wechselwirkung berücksichtigt. Eine Abschätzung des Einflusses der Coulomb-Abstoßung ist aus den Resultaten einer Arbeit von MOREL ${ }^{7}$ möglich.

1 J. Bardeen, L. N. Cooper u. J. R. Schrieffer, Phys. Rev. 108, 1175 [1958].

2 D. M. Ginsberg, Phys. Rev. 136, A 1167 [1964]. - R. I. GAYley, Phys. Letters 13, 278 [1964].

${ }^{3}$ R. E. Glover u. M. C. Sherrill, Phys. Rev. Leters 5, 248 [1960].

4 W. RüHL, Z. Phys. 159, 428 [1959] ; 186, 190 [1965] ; 196, 464 [1966].
Er hat den Phononen- und den Coulomb-Anteil der mittleren Wechselwirkung für ein parabelförmiges Elektronenband in Abhängigkeit von dem Parameter

$$
a^{2}=\left(k_{\mathrm{s}} / 2 k_{\mathrm{F}}\right)^{2}
$$

für eine Reihe einfacher Supraleiter berechnet. Dabei ist

$$
k_{\mathrm{s}}{ }^{2}=4 \pi e^{2} D\left(\varepsilon_{\mathrm{F}}\right)
$$

das Quadrat der reziproken Thomas-Fermi-Abschirmlänge, $e$ die Elektronenladung und $k_{\mathrm{F}}$ der Radius der Fermi-Kugel. In Abb. 1 ist die sich aus den Rechnungen ergebende Abhängigkeit der Kopplungskonstanten $g$ von $a^{2}$ für die von MOREL benutzten Supraleiter an-

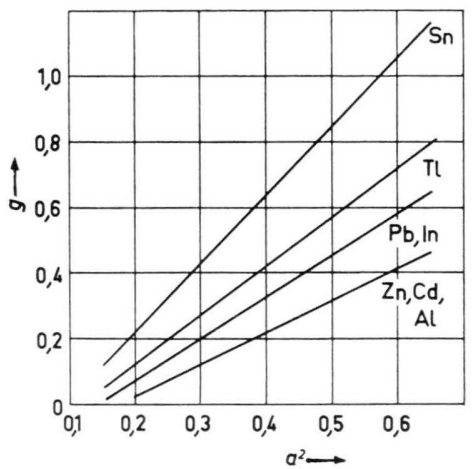

Abb. 1. Abhängigkeit der Kopplungskonstanten $g$ von dem in (3) definierten Parameter $a^{2}$ für eine Reihe einfacher Supraleiter. In dem hier interessierenden Bereich kann der Zusammenhang zwischen $g$ und $a^{2}$ durch die Gleichung $g=g_{0}+\gamma a^{2}$ analytisch dargestellt werden.

gegeben. Für den Vergleich mit den Experimenten ist es nun wichtig, daß die Morelschen Überlegungen in gleicher Weise für ein parabelförmiges Löcherband gelten. Für eine parabolische Näherung der Elektronenenergie

$$
\varepsilon(k)=\varepsilon_{0} \pm \frac{\hbar^{2} k^{2}}{2 m^{*}}
$$

( $\varepsilon_{0}$ ist die Energie der Bandkante, $m^{*}$ ist die effektive Masse des Bandes) hängt nun die Zustandsdichte an der Fermi-Kante mit dem Radius der Fermi-Kugel über

$$
D\left(\varepsilon_{\mathrm{F}}\right)=\frac{2 m^{*}}{(2 \pi \hbar)^{2}} k_{\mathrm{F}}
$$

zusammen. Daher ist die Kopplungskonstante auch in dieser Näherung eine abnehmende Funktion der $\mathrm{Zu}$ standsdichte, genauer $g=\alpha D\left(\varepsilon_{\mathrm{F}}\right)^{-1}+g_{0}$ (s. Abb. 1).

5 W. RüHL, Tagungsbuch der Sommerschule für Supraleitung der DPG, Steibis 1967, S. 127.

6 A. Bardasis, R. E. Prange u. E. D. Yorke, in Grundprobleme der Physik dünner Schichten, Göttingen 1965, S. 744. - A. Bardasis, R. E. Glover, R. E. Prange u. E. D. Yorke, Technical Report No. 659, University of Maryland 1967.

7 P. Morel, J. Phys. Chem. Solids 10, 277 [1959]. 


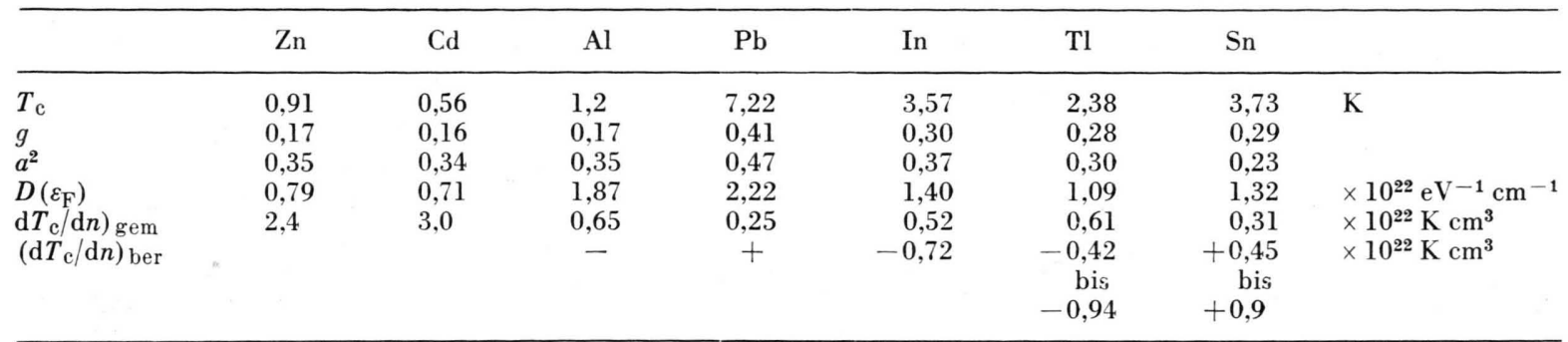

Tab. 1. Werte der Übergangstemperatur $T_{\mathrm{c}}$, der Kopplungskonstante $g$, des in (3) definierten Parameters $a^{2}$, der Zustandsdichte $D(\varepsilon \mathrm{F})$, der berechneten $\left(\mathrm{d} T_{\mathrm{c}} / \mathrm{d} n\right)$ ber und der gemessenen $\left(\mathrm{d} T_{\mathrm{c}} / \mathrm{d} n\right)$ gem Änderung der Übergangstemperatur bei Erhöhung der Leitungselektronendichte für einfache Supraleiter.

Durch Differentiation von (2) nach der Dichte $n$ der Leitungselektronen erhält man schließlich

$$
\frac{\mathrm{d} T_{\mathrm{c}}}{\mathrm{d} n}=\frac{1}{D} \frac{\mathrm{d} T_{\mathrm{c}}}{\mathrm{d} \varepsilon_{\mathrm{F}}}=-T_{\mathrm{c}} \frac{a^{2}}{g^{2}} \frac{\mathrm{d} g}{\mathrm{~d} a^{2}} \frac{\mathrm{d} \ln k_{\mathrm{F}}}{\mathrm{d} \varepsilon_{\mathrm{F}}},
$$

also die Verschiebung der Übergangstemperatur für jedes der Volumeneinheit hinzugefügte Elektron. Diese Größe kann für die in Abb. 1 angegebenen Supraleiter in der parabolischen Näherung berechnet werden (Tab. 1). Dabei sind die von Morel angegebenen Daten für $T_{\mathrm{c}}$ und $g$ eingetragen; $a^{2}$ ergibt sich mit den Werten von $g$ aus den Kurven von Abb. 1. Die Zustandsdichten $D\left(\varepsilon_{\mathrm{F}}\right)$ sind aus den von DAUNT ${ }^{8}$ mitgeteilten Elektronenanteilen der spezifischen Wärme ermittelt worden. Der nach Gl. (7) errechnete Betrag von $\mathrm{d} T_{\mathrm{c}} / \mathrm{d} n$ ist mit $\left(\mathrm{d} T_{\mathrm{c}} / \mathrm{d} n\right)_{\text {ber }}$ bezeichnet; er ist noch mit $\pm 1 \mathrm{zu}$ multiplizieren, je nachdem ob in (5) das positive oder negative Vorzeichen genommen wird. In der letzten Zeile stehen die experimentell gefundenen Werte von GLOVER und SHERRILL ${ }^{3}$ und RÜHL ${ }^{4}$. Während $\mathrm{Zn}$ und $\mathrm{Cd}$ durch direkten Elektronenentzug bisher noch nicht untersucht worden sind, ist für $\mathrm{Al}$ und $\mathrm{Pb}$ wenigstens auf das Vorzeichen von $\mathrm{d} T_{\mathrm{c}} / \mathrm{d} n, \mathrm{~d}$. h. die Richtung der $T_{\mathrm{c}^{-}}$ Verschiebung bei Erhöhung der Leitungselektronendichte aus Oxidationsversuchen zu schließen. Die ge-

8 J. G. Daunt, Progr. Low Temperature Physics, Vol. I, 1955, ed. by C. J. GORTER, S. 202.

9 J. R. ANderson u. A. V. Gold, Phys. Rev. 139, A 1459 [1965]. messenen Werte $\left(\mathrm{d} T_{\mathrm{c}} / \mathrm{d} n\right)_{\text {gem }}$ stimmen für $\mathrm{In}, \mathrm{Tl}$ und Sn trotz der einfachen parabolischen Näherung (5) für die Energiebandstruktur recht gut mit den berechneten überein. Nach (5) zeigt der Vorzeichenwechsel lediglich an, ob $\varepsilon_{\mathrm{F}}$ mehr in der Nähe einer unteren oder oberen Bandkante liegt. Nach den experimentellen Ergebnissen sollte dann bei den Metallen $\mathrm{Al}$, In und Tn überwiegend Elektronenleitung vorliegen, während für die Metalle $\mathrm{Pb}$ und $\mathrm{Sn}$ Löcherleitung anzunehmen wäre. Wegen der Abweichungen des Fermi-Körpers von der Kugelgestalt sind die Verhältnisse vermutlich komplizierter; benutzt man jedoch die von ANDERSON und GOLD $^{9}$ angegebene Zustandsdichtekurve für $\mathrm{Pb}$, so sollte sich das für $\mathrm{Pb}$ tatsächlich auch beobachtete positive Vorzeichen ergeben.

Bemerkenswert sind die errechneten Werte von $\mathrm{d} T_{\mathrm{c}} / \mathrm{d} n$ für die Metalle $\mathrm{Zn}$ und $\mathrm{Cd}$, die im wesentlichen auf einem gegenüber den übrigen Metallen sehr viel kleinerem Fermi-Körper beruhen. Ein solcher Unterschied ist von FARrell, PARK und Coles ${ }^{10}$ für $\mathrm{Zn}$ gefunden worden. Sie konnten an Hand von Legierungsexperimenten zeigen, daß der Einfluß der Änderung der Valenzelektronendichte auf die Sprungtemperatur bei $\mathrm{Zn}$ mehr als doppelt so groß ist wie bei In und $\mathrm{Sn}$.

10 D. Farrell, J. G. Park u. B. R. Coles, Phys. Rev. Letters 13. 328 [1964]. 